Artigo Original

Original Article

Claudia Regina Furquim de Andrade ${ }^{1}$ Fabiola Staróbole Juste ${ }^{1}$ Talita Maria Fortunato-Tavares ${ }^{1}$

Descritores

Gagueira

Criança

Priming

Tempo de reação

Linguagem

Fala

Métodos

Keywords

Stuttering

Child

Priming

Reaction time

Language

Speech

Methods

Endereço para correspondência: Claudia Regina Furquim de Andrade

R. Cipotânea, 51, Campus Cidade Universitária, São Paulo (SP), Brasil, CEP: 05360-160.

E-mail: clauan@usp.br

Recebido em: 8/2/2012

Aceito em: 6/9/2012

\section{Priming lexical em crianças fluentes e com gagueira do desenvolvimento}

\author{
Lexical priming in fluent and with developmental \\ stuttering children
}

\section{RESUMO}

Objetivo: Examinar a possível relação entre variáveis lexicais (categorização e nomeação) e gagueira do desenvolvimento. Métodos: Participaram do estudo 30 crianças falantes do português brasileiro, entre 7 a 9 anos e 11 meses. Foi utilizado o paradigma de priming lexical para investigar experimentalmente se as crianças com gagueira do desenvolvimento (Grupo Pesquisa) se diferenciam de seus pares fluentes (Grupo Controle), em relação ao tempo de reação em três condições de pesquisa - condição controle (sem prime); condição de prime semanticamente relacionado e condição de prime semanticamente independente em duas tarefas experimentais: categorização e nomeação do estímulo-alvo. Resultados: Na tarefa de categorização, não houve diferença no tempo de reação entre os grupos. O tempo de reação foi diferente para cada condição, porém, a variação foi similar em ambos os grupos. Houve efeito de prime entre as condições sem prime e prime relacionado indicando que, para ambos os grupos, o tempo de reação foi menor na condição de prime relacionado. Na tarefa de nomeação, houve diferença no tempo de reação entre os grupos. O tempo de reação no Grupo Pesquisa foi maior em relação ao Grupo Controle. Não houve efeito de prime, ou seja, em qualquer condição, o Grupo Pesquisa apresentou tempo de reação maior. Conclusões: Os resultados da pesquisa confirmaram a hipótese de que, nas crianças com gagueira do desenvolvimento, a prontidão na programação motora da fala - input - é lentificada, em relação ao Grupo de Controle de crianças fluentes. Não há diferença entre os grupos quando a função lexical não exige prontidão para a fala.

\begin{abstract}
Purpose: To examine the possible relationship between lexical variables (categorization and naming) and developmental stuttering. Methods: Thirty Brazilian Portuguese speaking children with ages ranging from 7 to 9 years and 11 months participated in the study. We applied a lexical priming paradigm to experimentally investigate whether children with developmental stuttering (Research Group) differed from their fluent peers (Control Group), with respect to reaction time in three conditions - control (without prime); semantically related prime, and semantically independent prime — of two experimental tasks: categorization and naming of the target stimulus. Results: No difference between groups was observed in reaction time on the categorization task. However, there was a condition effect showing that, for both groups, reaction time was shorter in the semantically related prime condition when compared to the no prime condition. In the naming task, a betweengroup difference was observed in reaction time, indicating a longer reaction time in the Research Group than the Control Group. There was no condition effect on naming, i.e. the Research Group showed slower reaction time regardless of prime type. Conclusions: The results confirm the hypothesis that, in children with developmental stuttering, readiness in motor programming of speech is slowed when compared to fluent children. There is no difference between groups when the lexical function does not require speech readiness.
\end{abstract}

Trabalho realizado no Departamento de Fisioterapia, Fonoaudiologia e Terapia Ocupacional, Faculdade de Medicina, Universidade de São Paulo - USP - São Paulo (SP), Brasil.

(1) Departamento de Fisioterapia, Fonoaudiologia e Terapia Ocupacional, Faculdade de Medicina, Universidade de São Paulo - USP - São Paulo (SP), Brasil.

Conflito de interesse: nada a declarar. 


\section{INTRODUÇÃO}

Segundo o paradigma do processamento motor da fala, a programação motora para $\mathrm{o}$ ato da fala gera a produção de movimentos sequenciais rápidos e suaves. As unidades de ação (conjunto organizado de comandos específicos e prontos para a ativação) são recuperadas da memória e utilizadas para cada situação motora específica. Embora os movimentos não sejam produzidos exatamente da mesma forma, sua características essenciais são mantidas porque os programas motores são generalizados e capturam os aspectos invariantes do movimento. $\mathrm{O}$ Programa Motor Generalizado (PMG) é um padrão de movimento que especifica quais músculos serão usados; qual a duração da ação e qual a força relativa das contrações musculares ${ }^{(1,2)}$.

A associação entre PMGs (da fala) e fatores do desenvolvimento (genéticos e ambientais) se integra de forma sinérgica com os demais sistemas responsáveis pela produção da fala (ex: respiratório; linguístico; psíquico, etc.). Os PMGs da fala correspondem aos comandos motores associados ao fonema; à sílaba; à palavra ou mesmo às sequências automáticas de fala (palavras produzidas frequentemente numa mesma sequência, chamada fala automática, como por exemplo: bom dia; como vai; contar números; falar os dias da semana, etc. $)^{(1,2)}$.

Baseado na meta do movimento (falar uma palavra de forma audível ao ouvinte) e nas condições disponíveis (nível de ruído ambiente, distância do ouvinte, a posição inicial da mandíbula), o PMG associado ao som alvo é complementado com os parâmetros disponibilizados no Esquema Motor (força muscular expiratória para a regulação da altura da emissão). As consequências sensoriais avaliam o resultado da produção e aceitam essa produção ou modificam o esquema motor numa nova tentativa ${ }^{(1,2)}$.

A abordagem da gagueira do desenvolvimento, dentro do modelo motor explicitado, busca justificar a ocorrência das rupturas involuntárias do fluxo da fala, sem possibilidade de recuperação espontânea, como sendo um déficit na programação motora da fala pelo comprometimento na geração do PMG. O déficit na programação motora da fala, pelo comprometimento na geração do PMG, pode decorrer das falhas organizacionais e temporais do movimento; pode decorrer das falhas na efetividade e adaptabilidade do esquema motor e/ou pelos efeitos de feedback (auditivo e somatossensorial $)^{(1,2)}$.

A hipótese da gagueira do desenvolvimento ser decorrente dos déficits na programação motora da fala (pelo comprometimento na geração do PMG) explicaria a diferença encontrada na performance de fala dos indivíduos com gagueira nas situações autoexpressivas (sujeita às rupturas, pois é gerada espontaneamente, exigindo capacidade para adaptação às mudanças súbitas e frequentes dos padrões motores) e nas situações de fala automática (quase sempre fluente pois é gerada dentro de PMGs já prontos e disponíveis $)^{(1-3)}$.

Dentro do modelo de programação motora da fala apresentado nos parágrafos anteriores existe uma vinculação intrínseca entre o processamento motor da fala e o processamento da linguagem (unidade motora e linguística operando paralelamente na preparação dos sinais de comando para a produção da fala). Durante a fase de aquisição e desenvolvimento da fala e da linguagem a operação moto/linguística está sujeita a ajustes constantes para a formação de mapas interativos eficientes (constantes e apurados). Essa vinculação intrínseca entre o processamento motor da fala e o processamento da linguagem tem sido pesquisada na gagueira do desenvolvimento, principalmente na população infantil ${ }^{(4-8)}$.

Dentre as possíveis vertentes de investigação da vinculação intrínseca entre o processamento motor da fala e o processamento da linguagem, a análise online tem se constituído um segmento de excelência para pesquisas dessa natureza pelo seu poder de captação de dados numa perspectiva dinâmica, mais próxima da fala usual ${ }^{(9)}$.

O paradigma experimental priming foi selecionado para o presente estudo por seu direcionamento para teste empírico das hipóteses relacionadas ao planejamento e processamento linguístico. O priming refere-se à mudança ou melhoria do desempenho do participante, quando desempenhando uma função cognitiva (por exemplo, tarefa de nomeação ou classificação lexical), como resultado da exposição a um estímulo ou a uma experiência anterior ${ }^{(10)}$.

O priming lexical envolve a noção de que um estímulo alvo (ex: uma figura) é processado e respondido, mais rápida ou precisamente, quando precedido por um prime semanticamente relacionado (ex: uma palavra de categoria semântica compatível com o estímulo alvo) do que quando comparado com um prime não semanticamente relacionado (ex: uma palavra de categoria semântica não compatível com o estímulo alvo). A apresentação do estímulo prime influencia o tempo de reação do participante. O tempo de reação será mais curto ou mais longo dependendo do tipo de prime utilizado. Se o prime e o estímulo-alvo forem semanticamente relacionados (por exemplo: prime auditivo = gato; figura-alvo $=$ cachorro), a codificação lexical será mais rápida, portanto o tempo de reação - tanto para classificar quanto para nomear o estímulo - será menor (em comparação com a condição de controle sem prime). Inversamente, se o prime e o estímulo alvo forem semanticamente independentes (por exemplo: prime auditivo = garfo; figura alvo $=$ cachorro), a codificação lexical será mais lenta — tanto para classificar quanto para nomear o estímulo - deverá ser mais lenta, portanto o tempo de reação será maior (em comparação com a condição de controle sem prime $)^{(10)}$.

A medida do tempo de reação (geralmente, realizada em escalas de milissegundos) permite ao pesquisador analisar o tempo aproximado do acesso lexical ou codificação de outros processos cognitivos do participante e, por conseguinte, a análise da velocidade de processamento em diferentes domínios linguísticos. A medida do tempo de reação é obtida por meio de programas computacionais precisos e específicos, como o E-Prime Experimental Control Software (PST, Inc.).

Estudos offline (pela aplicação de protocolos estruturados de avaliação da performance lexical) realizados em crianças com gagueira do desenvolvimento não são consensuais. Alguns estudos indicam que essas crianças com gagueira têm habilidades de codificação e organização lexicais menos desenvolvidas do que seus pares fluentes ${ }^{(9,11-15)}$. Outros estudos não encontram diferenças entre os grupos ${ }^{(16,17)}$. Estas discordâncias podem ser atribuídas a diferenças metodológicas tais como diferentes 
critérios de inclusão (como diagnóstico da gagueira e faixa etária) assim como diferentes materiais e tarefas utilizados.

Estudos do tempo de reação, em tarefa de priming lexical, podem contribuir para a ampliação do conhecimento sobre a influência das variáveis linguísticas na gagueira infantil ${ }^{(18)}$. O objetivo da pesquisa aqui apresentada é utilizar um paradigma de priming para acessar, experimentalmente, o comportamento de crianças que gaguejam em tarefas de categorização e nomeação lexical. A variável do tempo de reação foi analisada pela comparação dos resultados obtidos com crianças fluentes e com crianças com gagueira do desenvolvimento. Foram realizadas três condições de testagem (condição controle (sem prime); condição de prime semanticamente relacionado e condição de prime semanticamente independente) em duas tarefas distintas (categorização e nomeação do estímulo alvo).

\section{MÉTODOS}

\section{Considerações éticas}

O projeto foi aprovado pela Comissão de Ética em Pesquisa da Faculdade de Medicina da Universidade de São Paulo (USP), sob $\mathrm{n}^{\circ}$ 069/10. Todos os responsáveis assinaram o termo de consentimento livre e esclarecido. A pesquisa não envolveu técnica invasiva ou experimental não comprovadas, caracterizando o estudo como de baixo risco.

\section{Participantes}

Participaram do estudo 30 crianças falantes do português brasileiro entre 7 anos a 9 anos e 11 meses, de ambos os gêneros (sendo 22 meninos e 8 meninas, considerada a razão proporcional de incidência da gagueira $3.5 \hat{\jmath} / 1$ 우) e estrato socioeconômico cultural segundo meta-dados do IBGE de Classe C/D. As crianças foram divididas em dois grupos:

- Grupo Pesquisa (GP) - composto por 15 crianças com diagnóstico de gagueira do desenvolvimento há, no mínimo, 24 meses;

- Grupo Controle (GC) - composto por 15 crianças com desenvolvimento típico de linguagem, pareadas por gênero e idade ( \pm três meses) aos participantes do GP.

As crianças incluídas no GP foram recrutadas no Laboratório de Investigação Fonoaudiológica da Fluência, Funções da Face e Disfagia, do Departamento de Fisioterapia, Fonoaudiologia e Terapia Ocupacional da Faculdade de Medicina da Universidade de São Paulo. Crianças pertencentes ao GC foram recrutadas em escolas públicas da região metropolitana de São Paulo.

\section{Critérios de inclusão}

- Critérios de inclusão para ambos os grupos: ausência de comorbidades graves; ausência de desordem motora oral; ausência de dificuldades sociais e emocionais. Apresentar desempenho dentro da normalidade na Parte B (Vocabulário) do Teste de Linguagem Infantil
$\mathrm{ABFW}^{(19)}$. Apresentar limiares da audição dentro da normalidade;

- Critérios de inclusão em GP: apresentar grau de severidade de gagueira de no mínimo 2\% SLD - stuttering like disfluencies (rupturas gagas) por 100 sílabas $^{(20)}$;

- Critérios de inclusão em GC: não apresentar queixa nem histórico de qualquer distúrbio de linguagem. Apresentar pontuação dentro da normalidade na prova de fluência do Teste de Linguagem Infantil ABFW ${ }^{(19)}$.

\section{Materiais}

O experimento foi desenvolvido e apresentado por meio do E-Prime Experimental Control Software (PST, Inc.). A sequência e ordenação da apresentação das figuras-alvo e do prime auditivo, bem como a coleta e análise do tempo de reação, foram realizadas, randomizadas e controladas pelo E Prime Software (PST, Inc.). Os primes auditivos foram apresentados através de alto-falantes do notebook utilizado (Marca DELL SRS Premium Sound) com o volume controlado na mesma intensidade para todos os sujeitos de ambos os grupos. As respostas dos participantes foram capturadas através da caixa de respostas (response box da PST, Inc.) para a etapa de categorização. A captação das respostas de nomeação foi feita através de um microfone unidirecional (modelo MB-3K, da Audio-technica ${ }^{\circledR}$ ). Os dados de priming obtidos foram analisados pelo $E$ Merge software (PST, Inc.). As planilhas de dados foram montadas no Microsoft Office Excel 2007. A análise estatística foi realizada pelo software Statistica 8 (StatSoft, Inc.).

\section{Procedimentos}

\section{Condições de priming lexical}

Para coletar informações relativas ao tempo de reação durante as três condições - condição controle (sem prime); condição de prime semanticamente relacionado e condição de prime semanticamente independente - foi desenvolvido, pelas autoras do estudo, um experimento de nomeação e classificação lexical de figuras no E-Prime Experimental Control Software (PST, Inc.). As três condições de categorização de figuras foram empregadas em ordem contrabalanceada a todos os participantes de acordo com o método de estudo publicado anteriormente ${ }^{(18)}$ :

- Sem prime, na qual nenhum estímulo auditivo foi apresentado antes da exibição da imagem;

- Prime semanticamente relacionado, na qual uma palavra semanticamente relacionada à figura alvo foi apresentada auditivamente $700 \mathrm{~ms}$ antes da apresentação da figura na tela do computador;

- Prime semanticamente independente, na qual uma palavra não semanticamente relacionada foi apresentada auditivamente $700 \mathrm{~ms}$ antes da apresentação da figura na tela do computador.

Um intervalo de $700 \mathrm{~ms}$ foi aplicado após o início da apresentação do estímulo auditivo para garantir que nenhum prime auditivo fosse sobreposto ao onset visual da figura alvo. 


\section{Categorização e nomeação do estímulo alvo}

Para a tarefa de nomeação, cada participante foi apresentado a um conjunto de 27 figuras em cada uma das três diferentes condições (não prime, prime relacionado e prime independente). Para a tarefa de categorização, cada participante foi apresentado a um conjunto de 27 figuras (diferentes das apresentadas na tarefa de nomeação) em cada uma das três diferentes condições (não prime, prime relacionado e prime independente) (Tabela 1).

Tabela 1. Média (desvio padrão) da idade, pontuação de designação verbal usual (vocabulário) e \% de stuttering like disfluencies (fluência) para Grupo pesquisa e Grupo controle

\begin{tabular}{lcc}
\hline Variáveis & GP & GC \\
& Média (DP) & Média (DP) \\
\hline Idade & $9,3(1,4)$ & $9,6(1,3)$ \\
DVU (vocabulário) & $85,1(5,1)$ & $86,5(3,2)$ \\
$\%$ SLD (fluência) & $7,8(5,9)$ & $1,1(1,7)$ \\
\hline
\end{tabular}

Legenda: $\mathrm{GP}$ = grupo pesquisa; GC = grupo controle; $\mathrm{DP}=$ desvio padrão; DVU = designação verbal usual; SLD = stuttering like disfluencies (rupturas gagas)

As 54 figuras foram selecionadas a partir do conjunto de 118 figuras da Parte B do ABFW Teste de Linguagem Infantil(19) por amostragem aleatória simples (tabela de números aleatórios). Para controle de possíveis efeitos de experiência relacionados à figura, o teste $\mathrm{ABFW}$ foi aplicado somente após a coleta de dados do experimento. E, para evitar possíveis efeitos de experiência relacionados à condição de prime, ordem de apresentação das imagens dentro de uma condição foi randomizada, pelo sistema disponível no E-Prime.

Os primes auditivos foram gravados por uma falante nativa do português brasileiro. Os estímulos auditivos foram digitalizados e normalizados pelo software Praat.

\section{Procedimento geral e medidas de tempo de reação}

Todos os participantes realizaram as tarefas sentados numa cadeira confortável (com ajuste específico de altura), em frente a um computador a uma distância média de $30 \mathrm{~cm}$ da tela.

A instrução fornecida para a etapa de nomeação do experimento foi: "Figuras aparecerão na tela do computador. Diga o nome figura que aparecer na tela o mais rápido que você puder e assim que ela aparecer". O E-Prime software controlou a apresentação da figura alvo e do prime auditivo, assim como a latência de nomeação (tempo de reação de fala em milissegundos) medida a partir do onset da apresentação da figura alvo até o início desencadeamento da fala, pela resposta verbal do participante.

A instrução fornecida para a etapa de categorização do experimento foi: "Figuras aparecerão na tela do computador. Aperte 1 se a figura que aparecer no computador for "de comer" 2 se não for. $\mathrm{O}$ E-Prime software controlou a apresentação da figura alvo e do prime auditivo, assim como a latência de categorização (tempo de reação em milissegundos) medida a partir do onset da apresentação da figura alvo até a seleção da resposta do participante.

\section{RESULTADOS}

Somente as respostas corretas foram consideradas na análise do efeito de tempo de reação. Os três maiores e os três menores tempos de reação de cada participante em cada condição de prime foram excluídos. Na tarefa de nomeação, para o GP, só foram consideradas as produções fluentes para que não houvesse efeito da ruptura da fala na captação do tempo de reação. Somente uma produção de um dos participantes teve que ser excluída devido à apresentação de bloqueio na produção.

A análise estatística inferencial foi realizada por meio das análises de variância (ANOVA) de medidas repetidas e de um fator, para investigar diferenças em tempo de reação para as tarefas de categorização e nomeação entre crianças gagas e não gagas nas três condições de priming. A significância adotada foi de 5\%.

Ao realizar ANOVA de medidas repetidas, deve-se garantir que os dados assumam um critério de esfericidade, a qual presume que as variâncias das diferenças entre os dados de um mesmo participante em diferentes condições são iguais. O teste de Mauchly é utilizado para avaliar este critério. Se um teste de Mauchly é significativo $(\mathrm{p}<0.05)$, devemos concluir que há diferenças significativas entre as variâncias das diferenças e, portanto, o critério de esfericidade não é assumido. Neste caso, devemos corrigir os graus de liberdade da ANOVA para que esta forneça valores de F confiáveis. No presente estudo, como o teste de Mauchly foi significativo, foi aplicada a correção GreenhouseGeisser aos graus de liberdade da ANOVA de medidas repetidas.

\section{Tarefa de categorização}

A Figura 1 apresenta a média de tempo de reação (em milissegundos) e intervalo de confiança $(0,95)$ para as três condições de prime (não prime, prime independente e prime relacionado) para ambos os grupos na tarefa de categorização.

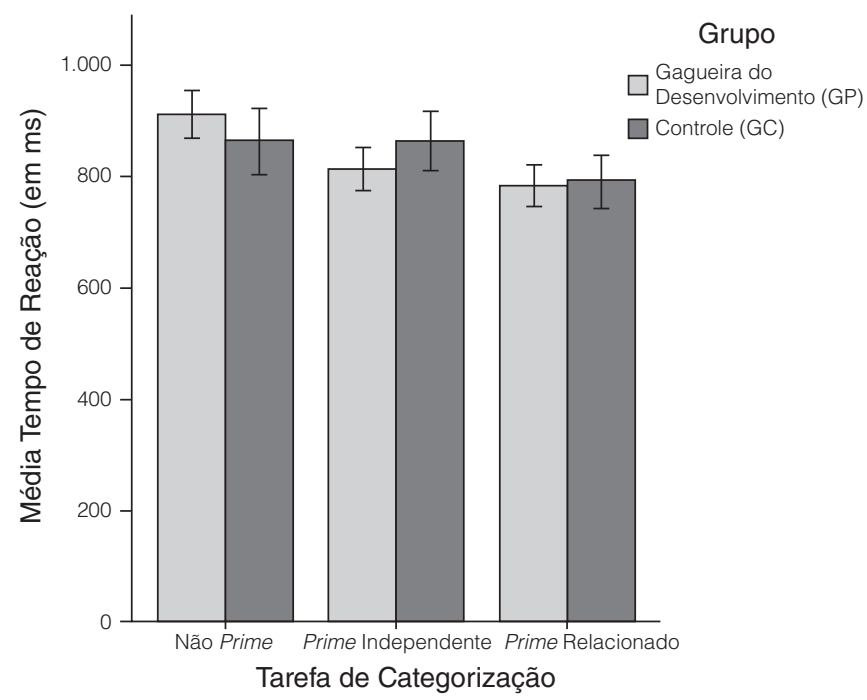

Figura 1. Médias de tempo de reação (em $\mathrm{ms}$ ) para a tarefa de categorização obtidas para o grupo controle e para o grupo pesquisa nas três diferentes condições de prime (não prime, prime independente e prime relacionado)

$\mathrm{Na}$ tarefa de categorização, o teste de Mauchly indicou que os dados assumem o critério de esfericidade (Mauchly's $\mathrm{W}=0,996 ; \mathrm{p}=0,431)$. Portanto, a ANOVA de medidas repetidas desta tarefa foi realizada sem a correção dos graus de liberdade.

Não houve interação significativa entre prime e grupo ( $F(2,944)=2,171 ; p=0,115)$ indicando que não há variação 
no tempo de reação entre os grupos nas diferentes condições de prime. Comparações a priori feitas com ANOVAs de um fator não revelaram diferenças entre os grupos de acordo com o tipo de prime (não prime: $\mathrm{F}(1,551)=2,612 ; \mathrm{p}=0,107$; prime independente: $\mathrm{F}(1,558)=1,959 ; \mathrm{p}=0,162$, prime relacionado: $\mathrm{F}(1,560)=0,665 ; \mathrm{p}=0,415)$.

Em ambos os grupos, os resultados revelaram um efeito significativo de condição de prime $(\mathrm{F}(2,944)=9,08 ; \mathrm{p}<0,001)$, indicando que o tempo de reação varia de acordo com o prime apresentado. Análises post hoc indicaram que há diferença entre a condição não prime e prime relacionado $(\mathrm{p}<0,001)$.

\section{Tarefa de nomeação}

A Figura 2 apresenta a média de tempo de reação (em milissegundos) para as três condições de prime (não prime, prime independente e prime relacionado) para ambos os grupos na tarefa de nomeação.

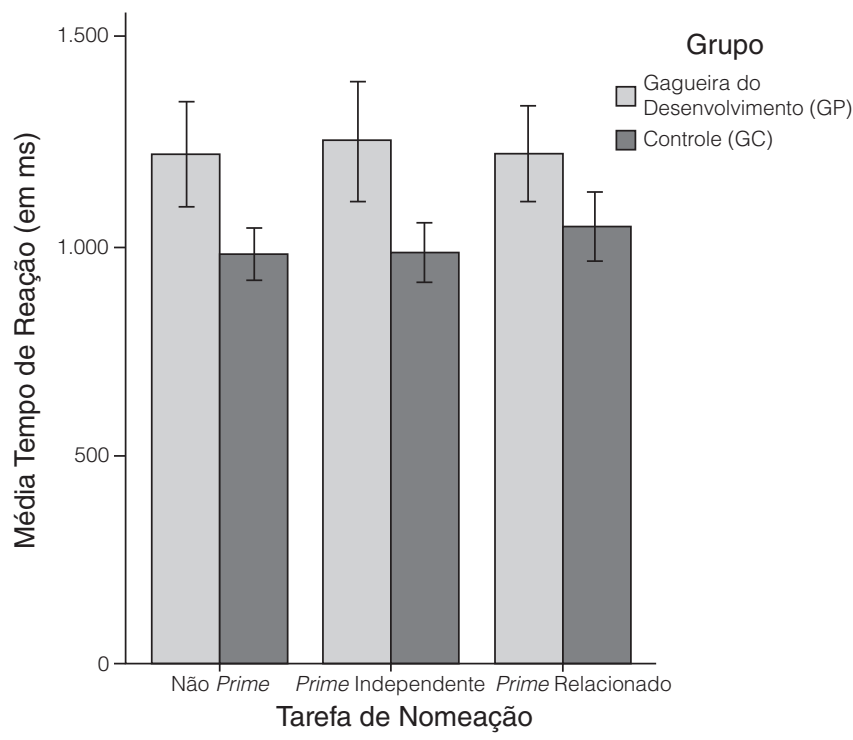

Figura 2. Médias de tempo de reação (em ms) para a tarefa de nomeação obtidas para o grupo controle e para o grupo pesquisa nas três diferentes condições de prime (não prime, prime independente e prime relacionado)

Na tarefa de nomeação, o teste de Mauchly indicou que os dados não assumem o critério de esfericidade (Mauchly's $\mathrm{W}=0,962 ; \mathrm{p}<0,001)$. Portanto, na ANOVA de medidas repetidas, os graus de liberdade foram corrigidos utilizando os estimadores de Greenhouse-Geisser.

A ANOVA de medidas repetidas não revelou interação significativa entre prime e grupo $(\mathrm{F}(1,926,941,974)=0,158$; $\mathrm{p}=0,846$ ). Entretanto, comparações a priori feitas por meio de ANOVAs de um fator revelaram diferenças entre os grupos para cada tipo de prime (não prime: $\mathrm{F}(1,571)=6.104 ; \mathrm{p}=0,014$; prime independente: $\mathrm{F}(1,569)=50,914 ; \mathrm{p}=0,015$; prime relacionado: $\mathrm{F}(1,560)=4,621 ; \mathrm{p}=0,032)$.

Os resultados não revelaram um efeito significativo de condição de prime $(\mathrm{F}(1,926,941,974)=0,080 ; \mathrm{p}=0,917)$, indicando que o tempo de reação não variou de acordo com o prime apresentado para nenhum dos grupos.

\section{DISCUSSÃO}

O presente estudo examinou a possível relação entre variáveis lexicais (categorização e nomeação) e gagueira do desenvolvimento. Trata-se de um estudo original, pois, para nosso conhecimento, até o momento são encontrados apenas estudos investigando a variável nomeação nesta população ${ }^{(18)}$. Foi utilizado o paradigma de priming lexical para investigar experimentalmente se as crianças com gagueira do desenvolvimento se diferenciam de seus pares fluentes em relação ao tempo de reação em três condições de pesquisa (condição controle (sem prime); condição de prime semanticamente relacionado e condição de prime semanticamente independente) nas tarefas de categorização e nomeação do estímulo alvo.

Os resultados indicam que, na tarefa de categorização, não há diferença no tempo de reação entre os grupos. O tempo de reação é diferente para cada condição, mas a variação é similar em ambos os grupos. Há efeito de prime entre as condições não prime e prime relacionado; isso significa que para ambos os grupos o tempo de reação é menor na condição de prime semanticamente relacionado.

Os resultados indicam que na tarefa de nomeação há diferença no tempo de reação entre os grupos. O tempo de reação no grupo de pesquisa é maior em relação ao GC. Não há efeito de prime, ou seja, em qualquer condição o GP apresenta tempo de reação maior.

\section{Efeito de tempo de reação quanto ao tipo de tarefa}

\section{Tarefa de categorização}

Os resultados do presente estudo indicaram que, na tarefa de categorização (atividade que não envolvia fala), não houve diferença no tempo de reação entre os grupos.

Não há um consenso na literatura sobre a relação entre acesso lexical e gagueira. Alguns autores ${ }^{(21,22)}$ sugerem que a gagueira possa refletir alguma perturbação no acesso lexical. Em contrapartida, outros estudos sugerem que a gagueira não está relacionada à dificuldade de recuperação lexical ${ }^{(23,24)}$, corroborando os resultados do presente estudo.

\section{Tarefa de nomeação}

Os resultados do presente estudo indicaram que o tempo de reação foi maior para o grupo com gagueira nesta tarefa (ou seja, parece haver um efeito lexical na gagueira somente quando a atividade motora da fala é recrutada). Este resultado corrobora estudo anteriormente realizados com crianças falante do inglês ${ }^{(9,14,15)}$ submetidas às mesmas condições de testagem ${ }^{(12,13,18)}$.

O tempo de reação aumentado para a fala em indivíduos com gagueira parece ser indicativo do impacto de uma falha na temporalização dos processamentos envolvidos na fala ${ }^{(7,8,19,22,25-27)}$. Os falantes com gagueira parecem não apresentar a mesma prontidão do sistema motor para a atividade de fala ${ }^{(25)}$. Esse déficit na prontidão para a fala pode ser decorrente desta falha na temporalização entre processamentos, sendo que esta se reflete em latências maiores para o início das atividades verbais, mesmo considerando as emissões fluentes dos falantes com gagueira ${ }^{(25)}$. 
Uma das teorias mais atuais sobre a gagueira, que considera a interação entre os sistemas linguístico e motor, é o modelo EXPLAN (derivado de EX - execução motora e PLAN, o mecanismo paralelo de planejamento linguístico) ${ }^{(26,27)}$. Os autores deste modelo consideram que o planejamento linguístico e a execução motora são processos que ocorrem paralelamente, porém, de maneira independente. Em razão da independência entre os processamentos (planejamento e execução), os mesmos podem ser escalonados e simultâneos, permitindo dessa forma, que um segmento possa ser planejado ao mesmo tempo que outro já está sendo executado. Essa concepção pode ser aplicada tanto para segmentos das frases (palavras) quanto aos segmentos das palavras (sílabas). Desta maneira, os autores sugerem que não existe erro no planejamento do segmento, o que pode ocorrer é que o mesmo ainda não esteja completamente pronto para a execução, ocorrendo desta forma, a falha na temporalização dos processamentos.

\section{Efeito de prime}

$\mathrm{Na}$ tarefa de categorização foi encontrado um efeito de prime; tempo de reação mais rápido para o para o prime relacionado se comparado ao prime neutro e o prime não relacionado para ambos os grupos. Este resultado encontra suporte teórico uma vez que o conceito de Priming Lexical considera que um estímulo alvo é processado e respondido mais rápida ou precisamente quando precedido por um prime semanticamente relacionado se comparado com um prime não semanticamente relacionado ${ }^{(28-30)}$.

$\mathrm{Na}$ tarefa de nomeação não foi encontrado um efeito de prime, o que contrasta com os achados de outro estudo ${ }^{(18)}$, no qual foi encontrado efeito de prime na nomeação tanto para o grupo de crianças gagas quanto para o grupo de crianças fluentes. Embora ambos os estudos tenham utilizado metodologia similar, duas variáveis podem ter contribuído para esta discondância. Em primeiro lugar, as faixas etárias dos participantes foram diferentes (no estudo americano crianças de 3 anos a 5 anos e 11 meses e em nosso estudo crianças de 7 a 9 anos e 11 meses). Em segundo lugar, este estudo envolveu o controle da variável de diagnóstico de gagueira do desenvolvimento com sintomatologia crônica há, no mínimo, 24 meses, variável não controlada no estudo anterior.

\section{CONCLUSÃO}

Os resultados da pesquisa, na forma que a mesma foi proposta, confirmam a hipótese de que nas crianças com gagueira do desenvolvimento a prontidão na programação motora da fala é lentificada em relação ao grupo controle de crianças fluentes. Não há diferença entre os grupos quando a função lexical não exige prontidão para a fala.

\section{AGRADECIMENTOS}

Esta pesquisa foi financiada pelo Conselho Nacional de Desenvolvimento Científico e Tecnológico (CNPq) (Edital Universal - processo $n^{\circ} 470458 / 2009-7$ ).
* CRFA foi responsável pelo projeto e delineamento do estudo e orientação geral das etapas de execução e elaboração do manuscrito; FSJ acompanhou a coleta e colaborou com a análise dos dados; TMFT foi responsável pela coleta e tabulação dos dados.

\section{REFERÊNCIAS}

1. Maassen B, Kent RD, Peters H, van Lieshout PHHM (eds). Speech Motor Control: Normal and Disordered Speech. Oxford: Oxford University Press; 2007.

2. Harrington J, Tabain M (eds). Speech Production: Models, Phonetic Process and Techniques. New York: Psychology Press; 2006.

3. Mildner V. The cognitive neuroscience of human communication. New York: Lawrence Erlbaum Associates; 2008.

4. Max L, Caruso AJ. Acoustic measures of temporal intervals across speaking rates: variability of syllable- and phrase-level relative timing. J Speech Lang Hear Res. 1997;40(5):1097-110.

5. Max L, Caruso AJ. Adaptation of stuttering frequency during repeated readings: associated changes in acoustic parameters of perceptually fluent speech. J Speech Lang Hear Res. 1998;41(6):1265-81.

6. Ingham R. On learning from speech-motor control research on stuttering. In: Cordes A, Ingham R (eds). Treatment efficacy for stuttering: A search for empirical bases. San Diego: Singular; 1998. p. 67-102.

7. Conture EG, Zackheim CT, Anderson JD, Pellowski MW. Linguistic processes and childhood stuttering: Many's a slip between intention and lip. In: Maassen B, Kent R, Peters H, van Lieshout P, Hulstijn W (eds). Speech motor control in normal and disordered speech. Oxford: Oxford University Press; 2004. p. 253-81.

8. Andrade CRF, Queiróz DP, Sassi FC. Eletromiografia e diadococinesia: estudo com crianças fluentes e com gagueira. Pro Fono. 2010;22(2):77-82.

9. Melnick KS, Conture EG, Ohde RN. Phonological priming in picture naming of young children who stutter. J Speech Lang Hear Res. 2003;46(6):1428-43.

10. McNamara TP, Holbrook JB. Semantic memory and priming. In: Weiner IB, Healy AF, Proctor RW (eds). Handbook of psychology: vol. 4 Experimental psychology. New York: Wiley; 2003. p. 447-74.

11. Anderson JD, Conture EG. Language abilities of children who stutter: A preliminary study. J Fluency Disord. 2000;25(4):283-304.

12. Anderson JD, Conture EG. Sentence structure priming in young children who do and do not stutter. J Speech Lang Hear Res. 2004;47(3):552-71.

13. Anderson JD. Age of acquisition and repetition priming effects on picture naming of children who do and do not stutter. J Fluency Disord. 2008;33(2):135-55.

14. Bishop JH, Williams HG, Cooper WA. Age and task complexity variables in motor performance of stuttering and nonstuttering children. J Fluency Disord. 1991;16(4):207-17.

15. Maske-Cash W, Curlee R. Effect of utterance length and meaningfulness on the speech initiation times of children who stutter and children who do not stutter. J Speech Hear Res. 1995;38(1):18-25.

16. Perozzi JA, Kunze LH. Language abilities of stuttering children. Folia Phoniatr (Basel). 1969;21(5):386-92.

17. Silverman S, Ratner NB. Measuring lexical diversity in children who stutter: Application of VOCD. J Fluency Disord. 2002;27(4):289-304.

18. Pellowski MW, Conture EG. Lexical priming in Picture naming of Young children who do and do not stutter. J Speech Lang Hear Res. 2005;48(2):278-94.

19. Andrade CRF, Befi-Lopes DM, Fernandes FD, Wetzner HF. ABFW: teste de linguagem infantil. 2 ed. São Paulo: Pro Fono; 2004.

20. Yairi E, Ambrose NG. Early childhood stuttering: for clinicians by clinicians. Austin: Pro/Ed; 2005.

21. Wingate ME. The structure of stuttering: A psycholinguistic analysis. New York: Springer-Verlag; 1988.

22. Weber-Fox C. Neural systems for sentence processing in stuttering. J Speech Lang Hear Res. 2001;44(4):814-25.

23. Onslow M, Packman A. Stuttering and lexical retrieval: Inconsistencies between theory and data. Clin Linguist Phon. 2002;16(4):295-8. 
24. Packman A, Onslow M, Coombes T, Goodwin A. Stuttering and lexical retrieval. Clin Linguist Phon. 2001;15(6):487-98.

25. Andrade CRF, Sassi FC, Juste FS, Meira MIM. Atividades de fala e nãofala em gagueira: estudo preliminar. Pro Fono. 2008;20(1):67-70.

26. Howell P, Dworzynski K. Planning and execution processes in speech control by fluent speakers and speakers who stutter. J Fluency Disord. 2005;30(4):343-54.

27. Savage C, Howell P. Lexical priming of function words and content words with children who do, and do not, stutter. J Commun Disord. 2008;41(6):459-84.
28. Velez M, Schwartz RG. Spoken word recognition in school age children with SLI: semantic, phonological, and repetition priming. J Speech Lang Hear Res. 2010;53(6):1616-28.

29. Girbau D, Schwartz RG. Implicit Semantic priming in spanish-speaking children and adults: an auditory lexical decision task. Span J Psychol. 2011;14(1):1-19.

30. Neely JH. Semantic priming and retrieval from lexical memory: roles of inhibitionless spreading activation and limited-capacity attention. J Exp Psychol Gen. 1977;106:226-54. 